\title{
THE BRAUER GROUP OF A REAL CURVE
}

\author{
F. R. DEMEYER AND M. A. KNUS
}

ABSTRACT. The Brauer group of a real algebraic affine curve is a finite group of exponent 2 whose rank is the number of connected real components (in the strong topology) of the curve.

Introduction. The purpose of this paper is to prove the following result.

THEOREM. Let $I$ be an ideal of coheight one in $\mathbf{R}\left[T_{1}, \ldots, T_{n}\right]$, the ring of polynomials in $n$ variables over the real numbers $\mathbf{R}$. Let $R=\mathbf{R}\left[T_{1}, \ldots, T_{n}\right] / I$ and let $X=\operatorname{Spec}(R)$ be the corresponding curve. If $s$ is the number of real components of $X$ and $\mathrm{Br}(R)$ denotes the Brauer group of $R$, then

$$
\mathrm{Br}(R) \cong(\mathbf{Z} / \mathbf{2 Z})^{s} .
$$

Hence the algebraic Brauer group of a real curve is the same as the topological Brauer group (see [3] for computations of topological Brauer groups).

The Brauer group of a complete nonsingular real curve was implicitely calculated by Witt [9] (see also [4]) and his result is the starting point for our proof. In $\$ 1$ we rephrase Witt's results. In $\$ 2$ the theorem is proved for irreducible nonsingular affine real curves. In $\$ 3$ the theorem is proved for irreducible affine curves and in $\$ 4$ arbitrary real affine curves are considered. In the course of the proof, we make some remarks on the Picard group and on higher cohomology groups of the curve. Finally $\$ 5$ gives the Brauer group of curves over finite fields.

While this paper was in preparation Walter Hill made several helpful suggestions. We have also taken into account some remarks of the referee which have improved the exposition. This paper was written while the first author visited at the Forschungsinstitut für Mathematik in Zurich. He thanks the officers of the Institut for their hospitability during his visit.

1. Complete nonsingular curves. Let $Y$ be a complete nonsingular real algebraic curve and let $K$ be its function field. Let $(\alpha, \beta)$ be the $K$-algebra defined by $u^{2}=\alpha, v^{2}=\beta, u v+v u=0, \alpha, \beta \in K$. By Tsen's theorem the Brauer group of $\bar{K}=K \otimes_{\mathbf{R}} \mathbf{C}$ is zero, hence any central simple $K$-algebra is equivalent to an algebra of the form $(\alpha,-1)$. The class of $(\alpha,-1)$ in $\operatorname{Br}(K)$ is trivial if and only if $\alpha=\gamma^{2}+\rho^{2}$ in $K$. Say that $\alpha$ is definite if $\alpha$ does not

Received by the editors September 8, 1975.

AMS (MOS) subject classifications (1970). Primary 13A20, 14F20, 14 H99.

C) American Mathematical Society 1976 
change sign on each connected component of $Y$. In [9] Witt proved the following (see also [4]):

(1.1). A function $\alpha \in K$ is positive definite (i.e. nonnegative on each real component) if and only if $\alpha=\gamma^{2}+\rho^{2}, \gamma, \rho \in K$.

(1.2). Choose a sign for each real connected component of $Y$, then there exists a function $\alpha$ on $Y$ taking values with these prescribed signs on the corresponding components. Call the corresponding algebras $(\alpha,-1)$ unramified.

(1.3). For each choice of an even number of points on each component of $Y$, there exists a function $\alpha$ on $Y$ changing signs at exactly the prescribed points.

It easily follows from (1.1)-(1.3) that the sequence

$$
0 \rightarrow(\mathbf{Z} / 2 \mathbf{Z})^{r} \rightarrow \mathrm{Br}(K) \stackrel{\tau}{\rightarrow} \bigoplus_{x}(\mathbf{Z} / 2 \mathbf{Z})_{x} \stackrel{\sigma}{\rightarrow}(\mathbf{Z} / 2 \mathbf{Z})^{r} \rightarrow 0
$$

is exact where $r$ is the number of real components of $Y$, the $x$ are the real points on $Y$, the map $\tau$ sends a class $(\alpha,-1)$ to an element in $\bigoplus_{x}(\mathbf{Z} / 2 \mathbf{Z})_{x}$ with nonzero entries at the points (necessarily even in number on each component) where $\alpha$ changes sign, the map $\sigma$ is the sum map on each component, and $(\mathbf{Z} / 2 \mathbf{Z})^{r}$ at the beginning of the sequence is the group of unramified classes in $\mathrm{Br}(K)$.

The first terms of the exact sequence (2.1) of Grothendieck in [5, III] are

$$
0 \rightarrow \mathrm{Br}(Y) \rightarrow \mathrm{Br}(K) \stackrel{\tau}{\rightarrow} \underset{x}{\oplus}(\mathbf{Z} / 2 \mathbf{Z})_{x}
$$

with $x$ and $\tau$ as in (1.4), so $\operatorname{Br}(Y)=(\mathbf{Z} / 2 Z)^{r}$ and if $A$ is an Azumaya algebra on $Y$, then the image of $A$ in $\operatorname{Br}(K)$ is an unramified algebra. Following [8, p. 229] we give an explicit construction of a representative of each class in $\operatorname{Br}(Y)$. Recall that an Azumaya algebra over a scheme $V$ with structure sheaf $O_{V}$ is a locally free sheaf of $O_{V}$-algebras on $V$ such that the geometric fiber $A(x)=A_{x} \otimes_{O_{x}} K(x)=A_{x} / m_{x} A_{x}$ is a central simple algebra over the field $K(x)=O_{x} / m_{x}$ for each point $x \in V$. The map $\operatorname{Br}(Y) \rightarrow \operatorname{Br}(K)$ is given by taking the fiber at the generic point. Let the image of $A$ in $\operatorname{Br}(K)$ be represented by $(\alpha,-1)$ where $\alpha$ is unramified. Now $\alpha$ is unramified if and only if the divisor $(\alpha)$ is the norm of a divisor on $\bar{Y}=Y \otimes_{R}$ C, i.e. $(\alpha)=\mathfrak{A}^{1+\rho}$ where $\rho$ is complex conjugation. Call a Zariski open set $U$ of $Y$ admissible if $\mathfrak{A}_{U}$ is principal. The admissible open sets form a basis for the Zariski topology, hence it suffices to define $A$ over admissible open sets. Let $\mathfrak{A}=(a), a \in K$, in an admissible open set $U$. Let

$$
\Gamma(U, A)=\Gamma\left(U, O_{\bar{Y}}\right) \oplus \Gamma\left(U, O_{\bar{Y}}\right) a^{-1} u \subset(\alpha,-1)
$$

where we think of $(\alpha,-1)$ as a crossed product $\bar{K} \oplus \bar{K} u, \bar{K}=K \otimes_{\mathbf{R}} \mathbf{C}$ and $u^{2}=\alpha$. This definition of $\Gamma(U, A)$ does not depend on the choice of $a$ since $\left.(a)\right|_{U}=\left.\left(a^{\prime}\right)\right|_{U} \Leftrightarrow a^{-1} a^{\prime} \in \Gamma\left(U, O_{Y}^{*}\right)$. It is clear that we obtain a locally free sheaf on the admissible open sets. As on p. 230 of [8] one can check that the multiplication in $(\alpha,-1)$ induces a multiplication in $\Gamma(U, A)$, that the fiber at the generic point is $(\alpha,-1)$ and that $A$ is central simple at each point $y \in Y$. Remark that if $A$ is an Azumaya algebra on $Y$ and if $Y_{1}, \ldots, Y_{r}$ are the real 
connected components of $Y$, then the map $A \mapsto\left(A\left(y_{1}\right), \ldots, A\left(y_{r}\right)\right)$ for any fixed choice $y_{i} \in Y_{i}$ induces the canonical isomorphism $\operatorname{Br}(Y) \cong(\mathbf{Z} / 2 \mathbf{Z})^{r}$. We shall employ this fact later.

Remark (1.6). Let $H^{n}\left(Y, G_{m}\right)$ be the etale cohomology of $Y$ with units as coefficients. It follows from the exact sequence (2.9) of [5, III] that

$$
\begin{aligned}
H^{2 n}\left(Y, G_{m}\right) & \cong H^{2}\left(Y, G_{m}\right) \cong \operatorname{Br}(Y) \text { for } n>0, \\
H^{2 n+1}\left(Y, G_{m}\right) & \cong \operatorname{Pic}(Y) / \operatorname{Norms}((\operatorname{Pic}(Y)) \text { for } n>0, \\
H^{1}\left(Y, G_{m}\right) & \cong \operatorname{Pic}(Y) .
\end{aligned}
$$

Remark (1.7). In [7] a pairing $\operatorname{Br}(Y) \times \operatorname{Pic}(Y) \rightarrow \operatorname{Br}(k)$ is constructed for complete nonsingular curves over any base field $k$. It was shown that for a $p$ adic field this pairing induces an isomorphism

$$
\operatorname{Br}(Y) \cong \operatorname{Hom}_{\mathbf{Z}}(\operatorname{Pic}(Y), \mathbf{Q} / \mathbf{Z}) .
$$

For real curves Witt showed Pic $(Y) / 2$ Pic $(Y)=(\mathbf{Z} / 2 \mathbf{Z})^{r}$ and the pairing gives the isomorphism $\operatorname{Br}(Y) \cong \operatorname{Hom}_{\mathbf{Z}}(\operatorname{Pic}(Y), \mathbf{Z} / 2 \mathbf{Z})$.

2. Irreducible nonsingular affine curves. Let $X=\operatorname{Spec}(R)$ be absolutely irreducible and let $Y$ be a regular completion of $X$ so that $Y-X$ contains only finitely many points. Let $Y_{i}, i=1, \ldots, r$, be the real components of $Y$ and suppose $X$ is obtained from $Y$ by deleting $n_{i}$ points from $Y_{i}$. Then $X$ has $s=\sum_{i} \max \left(1, n_{i}\right)$ real components. Suppose that for example $n_{1}=2$. Then by (1.2) and (1.3) there is a function which is not negative on $Y_{2}, \ldots, Y_{r}$ and which changes sign on the two parts of $Y_{1}$. Repeating the argument if necessary shows that for each choice of signs on the components of $X$ there is a definite function $\alpha \in K$ taking these prescribed signs. Now we conclude as in $\S 1$. If $X$ is irreducible but not absolutely irreducible, then $K$ contains a copy of $\mathbf{C}$. By Tsen's theorem $\operatorname{Br}(K)=0$ and hence $\operatorname{Br}(R)=0$. In this case $X$ has no real points so the theorem also holds.

Remark (2.1). The calculation for Pic gives Pic $(X) / 2$ Pic $(X)=(\mathbf{Z} / 2 Z)^{c}$ where $c$ is the number of closed components of $X$.

ReMARK (2.2). Comparing with Grothendieck's sequence (2.1) in [5, III], we also obtain that $\operatorname{ker}\left(H^{3}\left(X, G_{m}\right) \rightarrow H^{3}\left(K, G_{m}\right)\right)=(\mathbf{Z} / 2 \mathbf{Z})^{c}$ where $c$ is as in (2.1).

3. Irreducible affine curves. Let $X=\operatorname{Spec}(R)$ be an irreducible affine curve and let $R^{\prime}$ be the integral closure of $R$. Then $Y=\operatorname{Spec}\left(R^{\prime}\right)$ is an affine desingularization of $X$. Let $\mathrm{c}=\left\{x \in R \mid x R^{\prime} \subset R\right\}$ be the conductor. Since $R^{\prime} / \mathrm{c}$ is semilocal, the cartesian square

$$
\begin{aligned}
& R \rightarrow R^{\prime} \\
& \downarrow \quad \downarrow \\
& R / \mathfrak{c} \rightarrow R^{\prime} / \mathfrak{c}
\end{aligned}
$$

induces a Mayer-Vietoris sequence ([1] or [6]):

$$
0 \rightarrow \mathrm{Br}(R) \stackrel{\varphi}{\longrightarrow} \mathrm{Br}\left(R^{\prime}\right) \oplus \mathrm{Br}(R / \mathrm{c}) \stackrel{\psi}{\longrightarrow} \mathrm{Br}\left(R^{\prime} / \mathrm{c}\right)
$$


where $\varphi$ is the diagonal map and $\psi$ is the difference map. As usual, denote for any Azumaya algebra $A$ its Brauer class by $[A]$. By $\S 2$ we know that $\mathrm{Br}\left(R^{\prime}\right)=\mathrm{Br}(Y)=(\mathrm{Z} / 2 \mathrm{Z})^{r}$ where $r$ is the number of real components $Y_{1}, \ldots, Y_{r}$ of $Y$. More precisely, for each $j, j=1, \ldots, r$, there is an algebra $A_{j}$ on $Y$ such that $A_{i}\left(Y_{j}\right)=\delta_{i j}$ in $\mathrm{Br}(R)$ (this is meaningful since $A_{i}(y)$ $=A_{i}\left(y^{\prime}\right)$ if $y$ and $y^{\prime}$ are on the same component $\left.Y_{j}\right)$. These generators of the Brauer group of $Y$ will be used later in the proof of the theorem. Since

$$
R / \mathrm{c} \cong \mathbf{R}^{m} \oplus \mathbf{C}^{n}
$$

where the copies of $\mathbf{R}$ correspond to the real singular points $x_{1}, \ldots, x_{m}$ of $X$ and the copies of $\mathbf{C}$ to pairs of conjugate complex singular points, we obtain $\operatorname{Br}(R / \mathrm{c})=(\mathbf{Z} / 2 \mathbf{Z})^{m}$. For the following select algebras $B_{1}, \ldots, B_{m}$ over $R / \mathrm{c}$ such that $B_{i}\left(x_{j}\right)=\delta_{i j}$ in $\mathrm{Br}(R)$. Now decompose $R^{\prime} / \mathrm{c}$ according to (3.3). We have $R^{\prime} / \mathrm{c}=\bigoplus_{i=1}^{m} R_{i} \oplus \oplus_{i=1}^{n} C_{i}$ where the $R_{i}$ are Artin algebras over $\mathbf{R}$ and the $C_{i}$ Artin algebras over $\mathrm{C}$. We forget the $C_{i}$ since $\operatorname{Br}\left(C_{i}\right)=0, i=1, \ldots, n$. Decompose the $R_{i}$ further, $R_{i}=\bigoplus_{j} R_{i j}, 1 \leqslant j \leqslant l_{i}$, where $R_{i j}$ is indecomposable and

$$
R_{i j} / \operatorname{Rad}\left(R_{i j}\right)= \begin{cases}\mathbf{R} & \text { for } 1 \leqslant j \leqslant q_{i}, \\ \mathbf{C} & \text { for } q_{i}<j \leqslant l_{i} .\end{cases}
$$

Geometrically, to each $R_{i j}$ corresponds a point $y_{i j}$ on $Y$ lying over $x_{i}$ on $X$. The point is real if $1 \leqslant j \leqslant q_{i}$ and complex otherwise. Therefore we have $\operatorname{Br}\left(R^{\prime} / \mathrm{c}\right) \cong \bigoplus_{i=1}^{m}(\mathbf{Z} / 2 \mathbf{Z})^{q_{i}}$. In this sum there is a copy of $\mathbf{Z} / 2 \mathbf{Z}$ for each real point of $Y$ lying over a real singular point of $X$. Now we give a description of the map $\psi$ in our geometrical setting. As in $\S 1$ denote for any Azumaya algebra $A$ over a scheme $V$ the geometric fiber at a point $v \in V$ by $A(v)$. We first define $\psi$ on each summand. $\mathrm{On} \operatorname{Br}(Y)$ we have for the generators $A_{i}$,

$$
\psi\left(\left[A_{i}\right]\right)=\left[A_{i}\left(y_{k l}\right)\right]= \begin{cases}1 & \text { if } y_{k l} \in Y_{i}, \\ 0 & \text { if } y_{k l} \notin Y_{i} .\end{cases}
$$

On $\mathrm{Br}(R / \mathrm{c}) \psi$ is defined on the generators by

$$
\psi\left(\left[B_{i}\right]\right)=\left[B_{i}\left(y_{k l}\right)\right]= \begin{cases}1 & \text { if } y_{k l} \text { lies over } x_{i}, \\ 0 & \text { otherwise. }\end{cases}
$$

Finally $\psi$ can be defined on $\mathrm{Br}\left(R^{\prime}\right) \oplus \mathrm{Br}(R / \mathrm{c})$ as the sum (we have $\mathbf{Z} / 2 \mathbf{Z}$ modules!). We calculate $\mathrm{Br}(R)$ by computing ker $\psi$ in (3.2). Let $\left(\zeta_{1}, \ldots, \zeta_{r}\right.$, $\left.\xi_{1}, \ldots, \xi_{m}\right)$ be an element of $\operatorname{ker} \psi$, where the $\zeta_{i}$ are the components in $\operatorname{Br}(Y)$ and the $\xi_{j}$ the components in $\mathrm{Br}(R / \mathrm{c})$. If all $\zeta_{i}$ are zero, then we have $\xi_{j} \neq 0$, hence $\xi_{j}=\left[B_{j}\right]$ if and only if all the inverse images of $x_{j}$ on $Y$ are complex. In this way we obtain a subgroup $K_{1}$ of ker $\psi$ whose rank is the number of real singular points of $X$ with complex inverse images. Each such point is isolated (in the strong topology). If all $\xi_{j}$ are zero, then $\zeta_{i} \neq 0$, hence $\zeta_{i}=\left[A_{i}\right]$ if and only if the component $Y_{i}$ does not contain inverse images of real singular points. These components of $Y$ correspond to components of $X$ without real singular points. Therefore the kernel contains a subgroup $K_{2}$ whose rank is the number of components of $X$ without real singular points. Suppose now for example that the components $\zeta_{1}$ and $\xi_{1}$ of $\left(\zeta_{1}, \ldots, \xi_{1}, \ldots\right) \in \operatorname{ker} \psi$ are not zero. Suppose that the point $x_{1}$ lies on the component $X_{1}$ of $X$. Say now that 
$x_{1}, \ldots, x_{k}$ are the real singular points on $X_{1}$ and that $Y_{1}, \ldots, Y_{s}$ are the components of the inverse image of $X_{1}$. We claim that ker $\psi$ contains a diagonal subgroup of rank one whose generator is $\left(\left[A_{1}\right], \ldots,\left[A_{s}\right], 0, \ldots\right.$, $\left.\left[B_{1}\right], \ldots,\left[B_{k}\right], 0, \ldots\right)$. We have by assumption $\zeta_{1}=\left[A_{1}\right]$ and $\xi_{1}=\left[B_{1}\right]$. If $x_{2}$ has an inverse image on $Y_{1}$, then $\xi_{2} \neq 0$, hence $\xi_{2}=\left[B_{2}\right]$. If not, then say that $x_{2}$ has an inverse image on $Y_{2}$. Now notice that the inverse image of the component $X_{1}$ splits in real components $Y_{1}, \ldots, Y_{s}$ if and only if for each pair $Y_{\sigma}, Y_{\tau}, 1 \leqslant \sigma, \tau \leqslant s$, there is a sequence $Y_{\sigma}=Y_{\sigma_{1}}, \ldots, Y_{\sigma_{n}}=Y_{\tau}, 1 \leqslant \sigma_{l} \leqslant s$, and a sequence of singular points $x_{i_{1}}, \ldots, x_{i_{n-1}}$ on $X_{1}$ such that $x_{i_{j}}(1 \leqslant j<n)$ has inverse images on $Y_{j}$ and on $Y_{j+1}$. Applying the remark to the pair $Y_{1}, Y_{2}$ gives $\xi_{2} \neq 0$ and $\zeta_{2} \neq 0$, hence $\xi_{2}=\left[B_{2}\right]$ and $\zeta_{2}=\left[A_{2}\right]$. If $x_{2}$ has an inverse image on $Y_{2}$ and on another component, say $Y_{3}$, then clearly $\zeta_{3} \neq 0$, hence $\zeta_{3}=\left[A_{3}\right]$. The claim is proved by repeating the argument for all of the real singular points. In this way we obtain a diagonal subgroup of ker $\psi$ for each real component of $X$ containing real singular points with real inverse images. It is clear that $\operatorname{ker} \psi$ is the direct sum of all these diagonal subgroups and of $K_{1}$ and $K_{2}$. This sum is exactly $(\mathbf{Z} / 2 \mathbf{Z})^{s}$ with $s$ the number of real components of $X$.

Remark (3.4). Let $K$ be the quotient field of $R$. Since $\operatorname{Br}\left(R^{\prime}\right) \rightarrow \operatorname{Br}(K)$ is injective, we have also shown that $\operatorname{ker}(\operatorname{Br}(R) \rightarrow \operatorname{Br}(K))$ has rank equal to the number of real isolated points on $X$.

Remark (3.5). Formula (2.1) for Pic $(X) / 2$ Pic $(X)$ does not apply in general for singular curves. Let, for example, $R^{\prime}=\mathbf{R}[x, y]$ with $y^{2}=x^{4}$ -1 and $R=\mathbf{R}\left[y, x^{2}-1, x y, x\left(x^{2}-1\right)\right] \subset R^{\prime} ;$ then $\mathrm{c}=\left(y, x^{2}-1\right)$. The curve $y^{2}=x^{4}-1$ does not have closed components, hence Pic $\left(R^{\prime}\right) / 2$ Pic $\left(R^{\prime}\right)=0 \quad$ but $\quad \operatorname{Pic}(R)=U(\mathbf{R})$, hence Pic $(R) / 2$ Pic $(R)$ $=\mathbf{Z} / 2 \mathbf{Z}$. This follows from the Mayer-Vietoris sequence for Units and Pic. One gets that

$$
U\left(R^{\prime}\right) \oplus U(R / \mathrm{c}) \stackrel{\Phi}{\rightarrow} U\left(R^{\prime} / \mathrm{c}\right) \rightarrow \operatorname{Pic}(R) \rightarrow 0
$$

is exact. Now $U(R / \mathfrak{c})=U(\mathbf{R}), U\left(R^{\prime} / \mathfrak{c}\right)=U(\mathbf{R}) \oplus U(\mathbf{R})$, furthermore one verifies that the units of $R^{\prime}$ have same sign on both branches of $y^{2}=x^{4}-1$, hence the image of $\Phi$ is diagonal and Pic $(R)=U(\mathbf{R})$.

REMARK (3.6). The map $\psi$ in (3.2) is, in general, not surjective. Take, for example, the singular cubic $y^{2}=x^{2}(x+1)$; then Coker $\psi=\mathbf{Z} / 2 \mathbf{Z}$.

4. Affine curves. The final reduction to arbitrary affine curves employs the same technique as in $\$ 3$ and we shall not give details. Let $R=\mathbf{R}\left[T_{1}, \ldots, T_{n}\right] / I$ be the ring of the curve $X$. Since $\operatorname{Br}(R)=\operatorname{Br}\left(R_{\text {red }}\right)$ [2], we can suppose that $R$ is reduced. Then (0) is intersection of prime ideals, $(0)=\cap p_{i}$. Consider the canonical map $R \rightarrow S=\oplus_{i} R / \mathfrak{p}_{i}$. The conductor $\mathrm{c}$ from $S$ to $R$ is of the form $\mathrm{c}=\bigoplus_{i} \mathrm{c}_{i}$ where $\mathrm{c}_{i}=\operatorname{Ann}_{R} \mathfrak{p}_{i}=\cap_{j \neq i} \mathfrak{p}_{j}$. Applying the Mayer-Vietoris sequence to the cartesian square

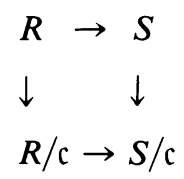

gives 


$$
0 \rightarrow \mathrm{Br}(R) \rightarrow \mathrm{Br}(S) \oplus \mathrm{Br}(R / \mathrm{c}) \stackrel{\psi}{\longrightarrow} \mathrm{Br}(S / \mathrm{c}) .
$$

The terms in the sequence have the following geometric interpretation: The rings $R / \mathfrak{p}_{i}$ are the affine rings of the irreducible components $X_{i}$ of $X$. The ideals $c_{i}$ contain all the elements of $R$ which vanish on the components $X_{j}$ for $j \neq i$. In $R / \mathfrak{p}_{i}$ the ideal $\mathfrak{c}_{i}$ is the intersection of maximal ideals corresponding to points on $X_{i}$ which also lie on some $X_{j}$ for $j \neq i$. Thus $\mathrm{c}$ is the intersection of maximal ideals corresponding to points of $X$ which lie on two or more irreducible components of $X$. Proceeding as in $\S 3$, one can check that ker $\psi$ has rank equal to the number of connected components of $X$.

5. Curves over finite fields. Sequence (2.1) of [5] and the Mayer-Vietoris sequence can also be easily applied to compute the Brauer group of an affine curve $X$ over a finite field. Let $Y$ be the normalization of $X$; then by MayerVietoris $\operatorname{Br}(X)=\operatorname{Br}(Y)$. Suppose now that $X$ is nonsingular and let $Y$ be a regular completion of $X$. By [5, Remarque 2.5(b)], $\mathrm{Br}(Y)=0$ and $H^{3}\left(Y, G_{m}\right)$ $=\mathbf{Q} / \mathbf{Z}$. It then follows from (2.1) of [5] that the sequence

$$
0 \rightarrow \operatorname{Br}(X) \rightarrow \underset{i}{\oplus}(\mathbf{Q} / \mathbf{Z})_{i} \rightarrow \mathbf{Q} / \mathbf{Z} \rightarrow 0
$$

is exact where the sum is taken over all points of $Y-X$.

\section{REFERENCES}

1. L. N. Childs, Mayer-Vietoris sequences and Brauer groups of nonnormal domains, Trans. Amer. Math. Soc. 196 (1974), 51-67.

2. F. R. DeMeyer, The Brauer group of a ring modulo an ideal, Rocky Mountain J. Math. 6 (1976), 191-198.

3. P. Donovan and M. Karoubi, Graded Brauer groups and K-theory with local coefficients, Inst. Hautes Études Sci. Publ. Math. 38 (1970), 5-25. MR 43 \#8075.

4. W.-D. Geyer, Ein algebraischer Beweis des Satzes von Weichold über reelle algebraische Funktionenkörper, Algebraische Zahlentheorie, Bericht Tagung Math. Forschungsinst., Oberwolfach, 1964, Bibliographisches Institut Mannheim, 1967, pp. 83-98. MR 37 \# 5224.

5. A. Grothendieck, Le groupe de Brauer. I, II, III, Dix Exposés sur la Cohomologie des Schémas, Masson, Paris; North-Holland, Amsterdam, 1968. MR 39 \#5586a, b, c.

6. M. A. Knus and M. Ojanguren, A Mayer-Vietoris sequence for the Brauer group, J. Pure Appl. Algebra 5 (1974), 345-360.

7. S. Lichtenbaum, Duality theorems for curves over p-adic fields, Invent. Math. 7 (1969), 120-136. MR 39 \#4158.

8. Ju. I. Manin, Cubic forms: Algebra, geometry, arithmetic, "Nauka", Moscow, 1972; English transl., North-Holland Math. Library, vol. 4, North-Holland, Amsterdam, 1973; American Elsevier, New York, 1974.

9. E. Witt, Zerlegung reeller algebraischer Funktionen in Quadrate, Schiefkörper über reellem Funktionenkörper, J. Reine Angew. Math. 171 (1934), 4-11. 80523

Department of Mathematics, Colorado State University, Fort Collins, Colorado Mathematik, E.T.H., CH-8006 Zurich, SwitzerLand 